CASE REPORT

\author{
P.O. Cowley \\ R. Jones \\ P. Tuch \\ W. McAuliffe
}

\section{Pulsatile Tinnitus from Reversal of Flow in an Aberrant Occipital Artery: Resolved after Carotid Artery Stenting}

\begin{abstract}
SUMmARY: Carotid artery stent placement, performed for correction of an asymptomatic severe stenosis, leads to the resolution of persistent and troublesome pulsatile tinnitus. Tinnitus has been reported as a consequence of severe carotid stenoses on previous occasions. This case highlights how an aberrant occipital artery originating above a carotid artery stenosis can result in flow reversal and be a mechanism by which tinnitus may develop.
\end{abstract}

$\mathbf{T}$ his case demonstrates that reversal of flow in the occipital artery may be an important cause of pulsatile tinnitus. Flow reversal in an aberrant occipital artery secondary to a carotid stenosis was rectified, leading to complete cessation of subjective and objective tinnitus.

\section{Case Report}

A 52-year-old woman was found to have a greater than 95\% stenosis of the right common carotid artery on sonography and on 2D timeof-flight MR angiography. The patient elected to have internal carotid artery (ICA) stent placement for correction of the severe stenosis. At no time did she have an ischemic event in any territory.

Of note, she also complained of persistent right-sided pulsatile tinnitus. There was a prominent and extensive bruit radiating from the neck up to the angle of the jaw, higher up to the tragus of the ear, and around the temporomandibular joint on the right side. It was not audible anyplace else on the skull. Compression of the internal jugular vein had no effect, excluding a venous hum. She had a cholesteatoma removed on that side in 1975, on a background of frequent otitis media. Since then, there had been frequent fungal infections in that area until 4 years before presentation. The relationship between the tinnitus and the stenosis was not clinically certain.

After antiplatelet medication (aspirin $150 \mathrm{mg}$ once daily and clopidogrel $75 \mathrm{mg}$ once daily), she then underwent angiography and proceeded directly to undergo endovascular stent placement. An apparent filling defect with streaming of unopacified blood in the ICA and absence of the occipital artery was noted on the preliminary images after common carotid artery injection (Figs 1 \& 2).

Using distal ICA protection (FilterWire EZ; Boston Scientific, Fremont, Calif), we placed an $8 \times 30$-mm nitinol self-expanding opencell stent (Precise; Cordis, Miami Lakes, Fla) across the lesion followed by 5 -mm angioplasty.

Repeated angiography after placement of the stent demonstrated filling of an aberrant large right occipital artery with its origin in the cervical right ICA (Figs $3 \& 4$ ). The patient stated that the tinnitus resolved at the time of stent placement.

Independent clinical auscultation confirmed complete resolution of the right-sided pulsatile tinnitus on the day of the procedure and

Received August 5, 2008; accepted after revision September 21

From the Department of Diagnostic and Therapeutic Neuroradiology and Interventional Services of Western Australia, Sir Charles Gairdner and Royal Perth Hospitals, Perth, WA, Australia.

Please address correspondence to Dr. Peter Cowley, DTNISWA, Sir Charles Gairdner Hospital, Nedlands, Perth, WA, Australia; e-mail: pedrocowley@gmail.com

DOI 10.3174/ajnr.A1393 demonstrated a sustained relief of symptoms 6 weeks after the procedure.

\section{Discussion}

Pulsatile tinnitus is a common presenting complaint that may arise from a host of possible underlying causes. The full list of differential diagnoses ${ }^{1}$ ranges from the benign to the impending lethal. A simplified scheme to review the causes of tinnitus considers 1) changes in flow velocity, 2) aberrant flow, and 3) turbulent flow. These 3 categories apply to both the arterial and the venous structures. Changes in flow velocity encompass causes such as dural arteriovenous fistulas and vascular tumors. Aberrant flow encompasses those such as high jugular bulb and persistent stapedial artery, and turbulence may be a contributing factor in any of the above causes and also in stenotic lesions of the carotid or transverse-sigmoid dural sinuses.

Reversal of flow and/or the possible changes in pulsatility and velocity within the occipital artery and its branches secondary to the carotid stenosis was the likely cause of the pulsatile tinnitus in the case of our patient. The close association of the occipital artery with the medial aspect of the mastoid process in the occipital groove makes it a good candidate for the propagation of abnormal pulsations to the structures of the middle and inner ear.

Pulsatile tinnitus has been attributed to many different vascular pathologic processes including stenotic lesions of the ICA. ${ }^{2,3}$ We cannot entirely exclude the possibility that the tinnitus was propagated directly from turbulence in the carotid stenosis. However, the character and distribution of the audible bruit that resolved after stent placement were not felt to be consistent with this explanation.

Many cases of pulsatile tinnitus secondary to dural arteriovenous fistulas involve abnormally high flow through the occipital artery. Furthermore, hypertrophy of the carotid to vertebral artery collateral vessels (the occipital artery in particular) has been linked to pulsatile tinnitus. Lehmann et $\mathrm{al}^{4}$ described such a case where subclavian stenosis lead to objective pulsatile tinnitus that resolved after subclavian artery stent placement.

Aberrant origins of the proximal branches of the external carotid artery have been described previously, including the ICA origin of the occipital artery. ${ }^{5-8}$ The exact incidence of this anatomic variant is unknown but is rare. Various series looking at the surgical anatomy of the carotid bifurcation have described the ICA origin of ascending pharyngeal, superior 


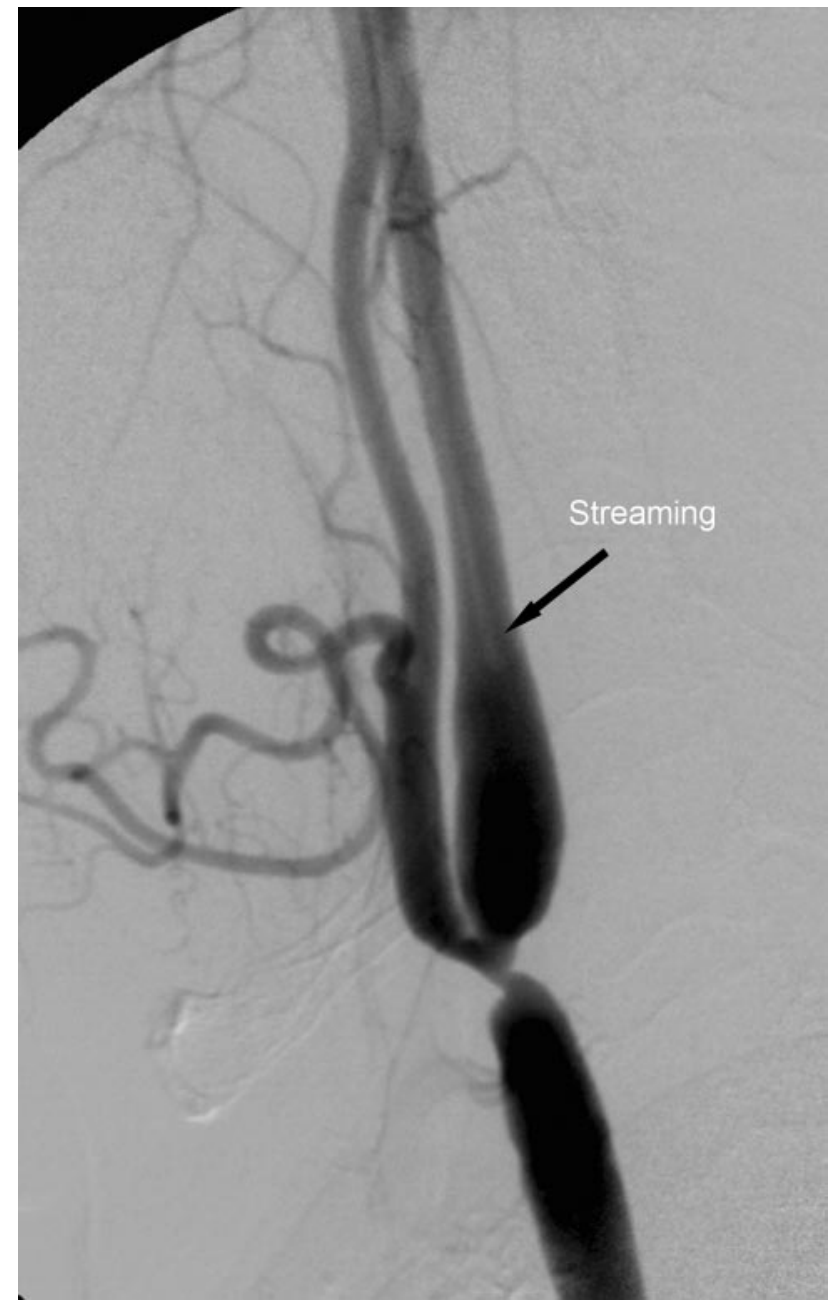

Fig 1. Lateral view of the right common carotid artery injection demonstrates severe stenosis at the bifurcation. Note streaming of unopacified blood within the ICA and the absence of occipital artery branch from the posterior surface of the external carotid artery.

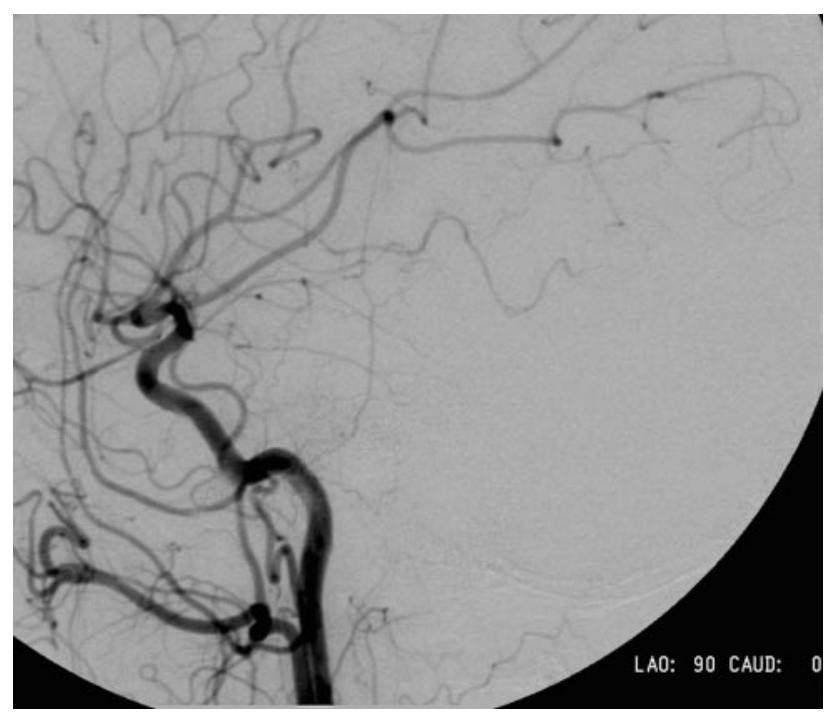

Fig 2. Lateral view of the right common carotid artery injection showing the apparent absence of the right occipital artery.

thyroid, lingual, and even facial arteries. Only 3 reports describing aberrant origin of the occipital artery have been found

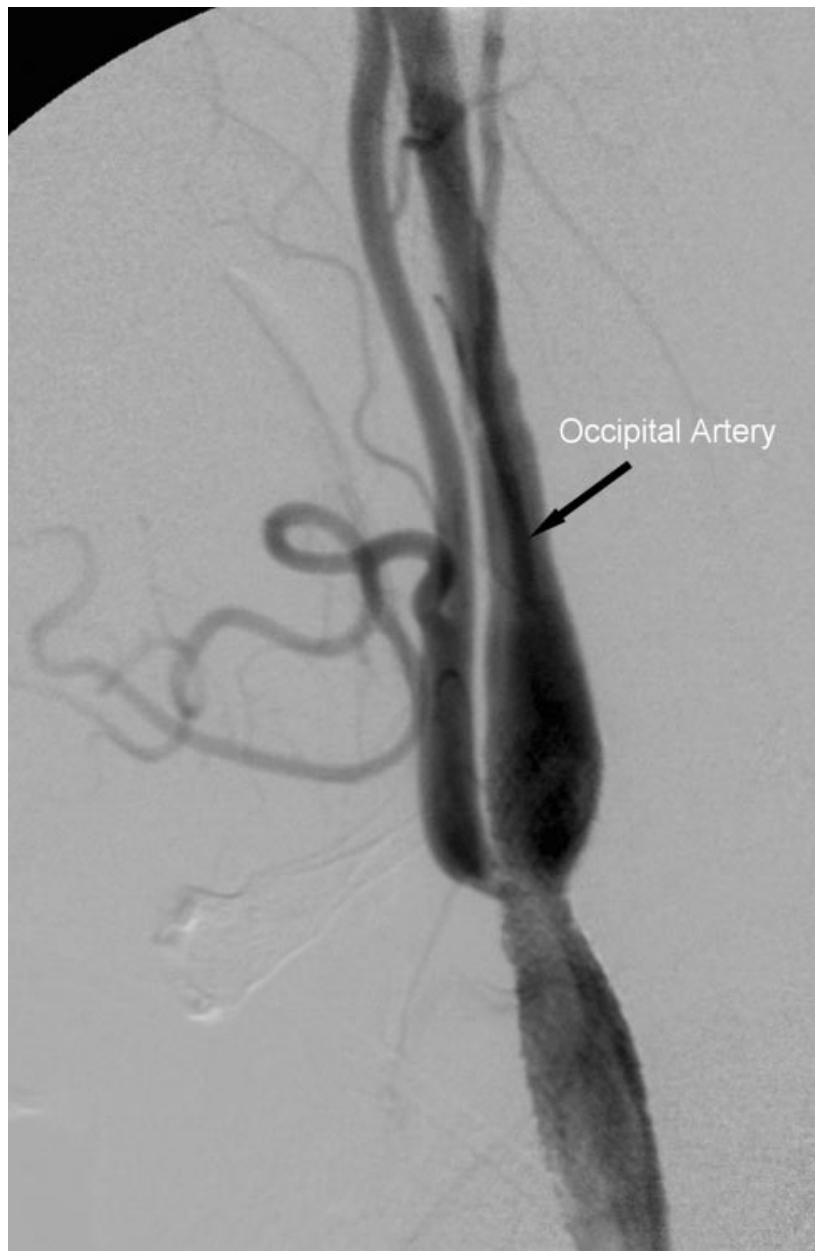

Fig 3. Lateral view of the right common carotid artery injection after stent placement. The previously unseen occipital artery is now opacified, taking its origin from the cervical ICA at the level of $\mathrm{C} 3$.

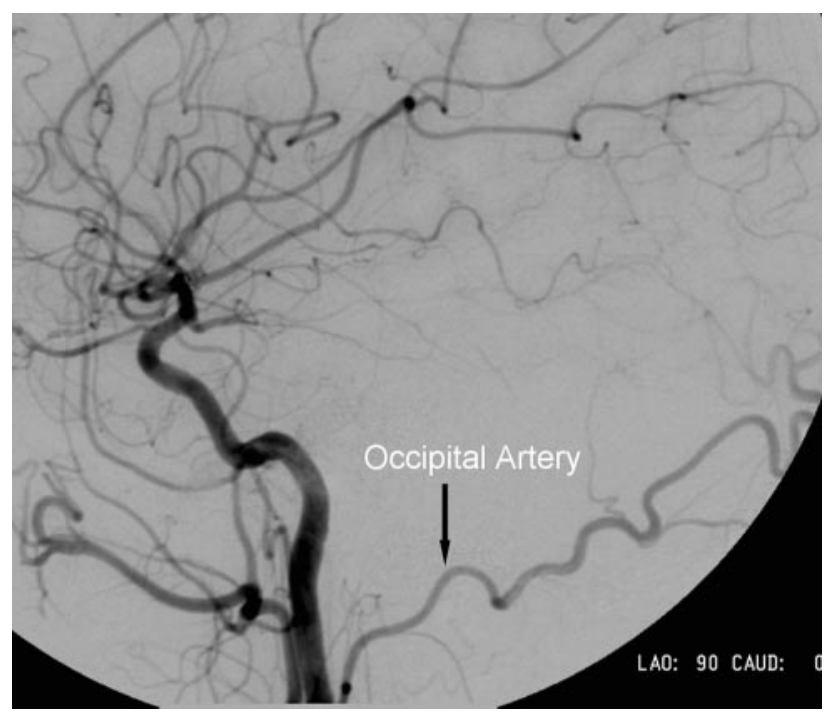

Fig 4. Lateral view of the right common carotid artery injection confirms the presence of the hitherto unseen occipital artery.

in the medical literature. ${ }^{5-7}$ The incidence of aberrant ICA origin of the occipital artery would seem to be considerably less than $1 \%$. 


\section{Conclusions}

We have presented a case that has never been described previously. In general, it highlights the potential role the occipital artery and, more specifically, reversal of flow within the occipital artery may play in the pathophysiology of pulsatile tinnitus.

\section{References}

1. Swartz JR, Harnsberger HR. Imaging of the Temporal Bone 3rd Ed. New York: Thieme; 1998:185

2. Waldvogel D, Mattle H, Sturzenegger M, et al. Pulsatile tinnitus - a review of 84 patients. J Neurol 1998;245:137-42

3. Emery D, Ferguson R, Williams JS. Pulsatile tinnitus cured by angioplasty and stenting of petrous carotid artery stenosis. Arch Otolaryngol Head Neck Surg 1998;124:460-61

4. Lehmann M, Mounayer C, Benndorf G, et al. Pulsatile tinnitus: a symptom of chronic subclavian artery occlusion. AJNR Am J Neuroradiol 2005;26:1960-63

5. Aggarwal NR, Krishnamoorthy T, Devasia B, et al. Variant origin thyroid artery, occipital artery and ascending pharyngeal artery from a common trunk from the cervical segment of internal carotid artery. Surg Radiol Anat 2006;28:650-53

6. Hachem K, Slaba S, Nassar J, et al. Imaging of an aberrant occipital artery arising from the extracranial segment of the internal carotid artery. J Maladies Vasculaires 2004;29:205-09

7. Ustunsoz B, Gumus B, Koksal A, et al. Missed total occlusion due to the occipital artery arising from the internal carotid artery. Cardiovasc Intervent Radiol 2007;30:116-17

8. Hayashi N, Hori E, Ohtani Y, et al. Surgical anatomy of the cervical carotid artery for carotid endarterectomy. Neurol Med Chir (Tokyo) 2005;45:25-30 